\title{
CD Imaging at High Spatial Resolution at Diamond B23 Beamline: Evolution and Applications
}

\author{
Rohanah Hussain, Tamás Jávorfi and Giuliano Siligardi * \\ Diamond Light Source Ltd., Harwell Science and Innovation Campus, Didcot, United Kingdom
}

Circular Dichroism imaging in the 190-650 nm spectral region pionered at Diamond Light Source B23 beamline, has been made possible by the highly collimated microbeam generated at the beamline and has been used to study the homogeneity of the supramolecular structures of thin films of chiral materials deposited on fused quartz substrates. This facility has been expanded with the installation of a Mueller Matrix Polarimeter, MMP, coupled to the beamlight, of which a preliminary data will be discussed. In the solid state, the measurement of CD related to the supramolecular structure is hampered by the presence of circular birefringence, linear dichroism, and linear birefringence that can only be evaluated using the MMP technique. The ability to characterize the chiroptical property of thin chiral films prepared under a variety of

OPEN ACCESS

Edited by:

Victor Borovkov,

Tallinn University of Technology,

Estonia

Reviewed by:

Bart Kahr

New York University, United States

Honghui He,

Tsinghua University, China

*Correspondence:

Giuliano Siligardi

giuliano.siligardi@diamond.ac.uk

Specialty section:

This article was submitted to

Supramolecular Chemistry,

a section of the journal

Frontiers in Chemistry

Received: 13 October 2020

Accepted: 16 February 2021

Published: 08 April 2021

Citation:

Hussain R, Jávorfi T and Siligardi $G$ (2021) CD Imaging at High Spatial Resolution at Diamond B23 Beamline:

Evolution and Applications.

Front. Chem. 9:616928.

doi: 10.3389/fchem.2021.616928 conditions and protocols such as drop cast, spin coating, spray at different temperatures and concentrations will enable the determination of the critical parameters for reproducible, uniform and homogeneous specimen preparation, which is the sine qua non for any commercial application. This is of particular importance for optoelectronic materials, but it can also be extended to a broad variety of materials with applications from biosensors to biological tissues.

Keywords: circular dichroism, CD imaging, Mueller matrix polarimetry, nano crystalline cellulose, supramolecular structure

\section{INTRODUCTION}

Optical activity is the property of a molecule in the solid state (Arago, 1811) and in solution (Biot, 1815) to rotate the plane of polarized light that passess through it (Biot, 1815).

Circular dichroism (CD) spectroscopy is the technique most often used to characterize optically acitive chiral molecules. A molecule is chiral if its mirror image forms are nonsuperposable (Pasteur, 1849), like those of $\mathrm{L}$ and $\mathrm{D}$ amino acids. In general, any entity that is dissymmetric, namely without a plane of symmetry or a center of inversion is chiral. This applies not only to molecules but materials made of large chiral polymers, combined achiral polymers doped with small chiral molecules, and achiral polymers adopting chiral architectures.

Optically active materials show $\mathrm{CD}$ that is the difference between the absorption of left and right circularly polarized light passing through the sample (Cotton, 1895).

In a chromophore of a chiral molecule, an electronic transition is an helical displacement of an electron charge induced differently by the left and right circularly polarized light. The quantummechanical model of the one-electron mechanism describes the helical displacement of charge generated by the magnetic dipole transition moment as the rotation of charge and the electric dipole transition momement as the linear displacement of charge. Another mechanism is the exciton 

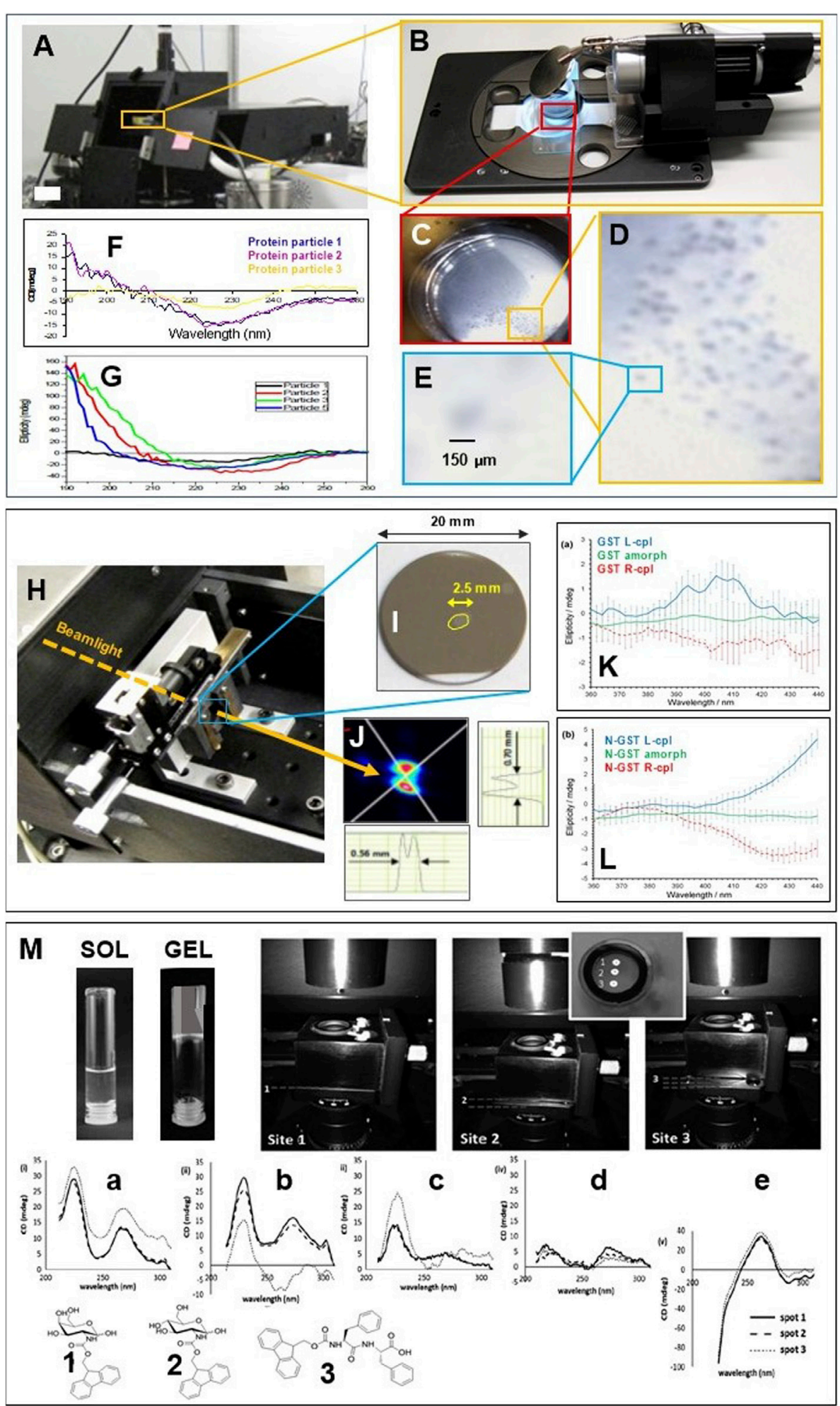

FIGURE 1 | (A) B23 vertical sample chamber of module A. (B) digital camera with mirror at $45^{\circ}$ to view the cuvette containing a suspension of protein particles in a physiological solution. (C, E) zommed images of cuvette cell, particles and single particle with bar size of $150 \mu \mathrm{m}$. (F) SRCD spectra of three selected particles of a $\beta$-sheet rich protein (Redrawn from 11). (G) a different preparation of the same proteins of (F-H) X-Y mechanical stage enables SRCD to be measured at different spots of the GST yellow circled area investigated using the vertical chamber of B23 module B. (I) Fused silica substrate window coated with a thin film of GST of which the spot of about $2.5 \mathrm{~mm}$ in length (circled in yellow for clarity) was sculpted with laser of left- or right-cpl. (J) Beamlight profile of about 600 $\mu \mathrm{m}$ in diameter at the GST 
FIGURE 1 | specimen measured with beam profile monitor Beamage USB. (K) SRCD spectra of GST sculpted with L-cpl (blue), R-cpl (red) and GST outside the spot in Figure 1I, the amorphous GST (green). (L) SRCD spectra of N doped GST sculpted with L-cpl (red), R-cpl (red) and amorphous N-GST (green). All figures redrawn from Borisenko et al. (2015). (M) SRCD spectra of five gel specimens prepared from three types of samples: (A) GalNHFmoc (1) hydrogel in water triggered thermally (0.01 cm cell); (B) GalNHFmoc hydrogel (1) in PBS triggered by sonication (0.02 cm cell); (C) GalNHFmoc (1) hydrogel in PBS triggered thermally (0.01 cm cell); (D) GlcNHFmoc (2) hydrogel in water triggered thermally (0.02 cm cell); and (E) Fmoc-FF (3) hydrogel in PBS triggered by sonication ( $0.01 \mathrm{~cm}$ cell). All samples were gelled in the cuvette cells at a concentration of $2.0 \mathrm{mg} \mathrm{ml}^{-1}$. For each specimen, three different positions were measured as illustrated in the insert [redrawn from Sitsanidis et al. (2018)].

coupling where the helical displacement of charge is generated by the coupling of electric dipole transition moments of two twisted chromophores of the chiral molecule framework (Mason, 1978).

The different velocity at which left and right circularly polarized light travels through chiral materials is called optical rotatory dispersion (ORD) and like the counterpart $\mathrm{CD}$ is a manifestation of the optical activity. ORD is generated by circular birefringence that is the difference in refractive index for left and right circularly polarized light (L-cpl and R-cpl) (Fresnel, 1824).

Modern CD instruments use one photoelastic modulator (PEM) to modulate the retardation of the incident linearly polarized light, of which only the quarter wave retardation is selected (Grosjean and Legrand, 1960; Velluz et al., 1965) and measured by a photomultiplier tube (PMT) or an avalanche photo diode (APD) detector (Hussain et al., 2012). The measurement of ORD is achieved by inserting in the CD spectropolarimeter a polarizing prism, the analyser, in between the sample, and the light detector (Lowry, 1935).

The highly collimated micro beamlight generated at B23 beamline for synchroton radiation circular dichroism (SRCD) (Hussain et al., 2012) enabled the measurements of CD of solid state protein microparticles to assess the conformation homogeneity of their preparation. To our knowledge, that was the first time that CD of individual particles of $150 \mu \mathrm{m}$ in diameter of a $\beta$-sheet rich protein was successfully measured without spectral artifacts due to light scattering and birefringence contributions (Figures 1A-G) (Gerdova, 2013). To identify and irradiate the single particles with the beamlight (Figures 1D,E), a $45^{\circ}$ mirror to deflect the beamlight to a digital camera orthogonal to the incident light (Figure 1B) demonstrated the feasibility of this method. As the activity of proteins is strictly related to their active conformation, the production of proteins in different foldings than the active one is one of the main causes of reducing the overall protein function and activity. The ability to monitor the protein conformation under a variety of enviromental conditions is of paramount importance. It enables the optimization of the protein formulation parameters to obtain reproducible protein particles of retained native activity associated to beta strand-rich folding as illustrated in the example of the particles batch of Figure 1F compared to that in Figure 1G where significant spectral differences were observed for the same protein particles but prepared with a different method. This proof-of-principle experiment pave the way to expand and extend this approach to many other chiral materials with various applications from Life Science to Physical Sciences.

The use of an X-Y manual stage enabled the measurements of SRCD at different spots of about $600 \mu \mathrm{m}$ in diameter of thin solid state films of pure $\mathrm{Ge}_{2} \mathrm{Sb}_{2} \mathrm{Te}_{5}$ (GST) and N-doped $\mathrm{Ge}_{2} \mathrm{Sb}_{2} \mathrm{Te}_{5} \mathrm{~N}$
(N-GST) phase-change memory materials coated on fused silica substrate (Figures 1H,J; Borisenko et al., 2015). An area of about $2.5 \mathrm{~mm}$ in diameter of amorphous pure and N-doped GST wafer of $22 \mathrm{~mm}$ in diameter was pre-irradiated with L-cpl and R-cpl respectively using a powereful laser (Figure 1H) that induced a permanent optical activity of opposite chirality (Figures $1 \mathbf{K}, \mathbf{L}$ ) due to rapid photo-crystallization of achiral amorphous GST. The sculpted pure or N-doped achiral GST materials with L-cpl or $\mathrm{R}$-cpl laser irradiation has been suggested as a potential method to control the induction of anisotropic optical properties for optoelectronic and photonic applications (Borisenko et al., 2015).

The self-assembly of three different peptide-based compounds, GalNHFmoc ( 1 in Figure 1M), GlcNHFmoc (2 in Figure 1M), and Fmoc-FF ( 3 in Figure 1M) from sol to gel state was for the first time investigated using the B23 vertical chamber developed for high throughput CD (HTCD) (Hussain et al., 2016) to probe the homogeneity and uniformity of the supramolecular structure of the three hydrogel materials (Sitsanidis et al., 2018) of different adjacent spots of about $100 \mu \mathrm{m}$ size. This was achieved by using a $10 \times$ objective lens that focused the beamlight to about $100 \mu \mathrm{m}$ in diameter on the sample without creating spectral distortions. The gelation of the peptide compounds form sol state in cylindrical cuvette cells was triggered by two methods: sonication and thermal heating and cooling cycles (Figure 1M). In an iterative manner, the method enabled the scientists to induce the gelation by varying one parameter per time in order to find out the optimum protocol for a reproducible homogeneous self-assembling. The benchtop CD instruments would give an average of a much larger area $(2-3 \mathrm{~mm}$ in diameter) than that with B23.

The use of a $10 \times$ objective lenses transparent down to $190 \mathrm{~nm}$ and a motorized $\mathrm{X}-\mathrm{Y}$ stage in the $\mathrm{B} 23$ vertical chamber enabled the $\mathrm{CD}$ imaging $(\mathrm{CDi})$ of thin films of chiral materials at high spatial resolution unattainable with bespoke benchtop $C D$ instruments or any other SRCD beamline. The use of B23 CDi tower was first exploited by the $\mathrm{Di}$ Bari group for the characterization of the local supramolecular order in thin films of chiral functional conjugated polymers with optoelectronic properties (Zinna et al., 2017).

A spatial resolution of $100 \mu \mathrm{m}$ revealed an inhomogeneous supramolecular structure of the thin film of chiral 1,4-phenylenebased oligothiophene polymer (Albano et al., 2019) (Figures $\mathbf{2 A - H ) . ~ T h e ~ S R C D ~ s p e c t r a ~ o f ~ t h a t ~ f i l m ~ m e a s u r e d ~ i n ~ b a c k ~ s i d e ~}$ orientation showed opposite sign than the front side that was indicative of linear dichroism (LD) and linear birefringence (LB) contributions to $\mathrm{CD}$ and circular birefringence $(\mathrm{CB})$ (Figures 2A,B). The fact that the SRCD spectral profiles of both front and back side orientations decreased in overall intensity as a function of time was consistent with a rearrangement of the 

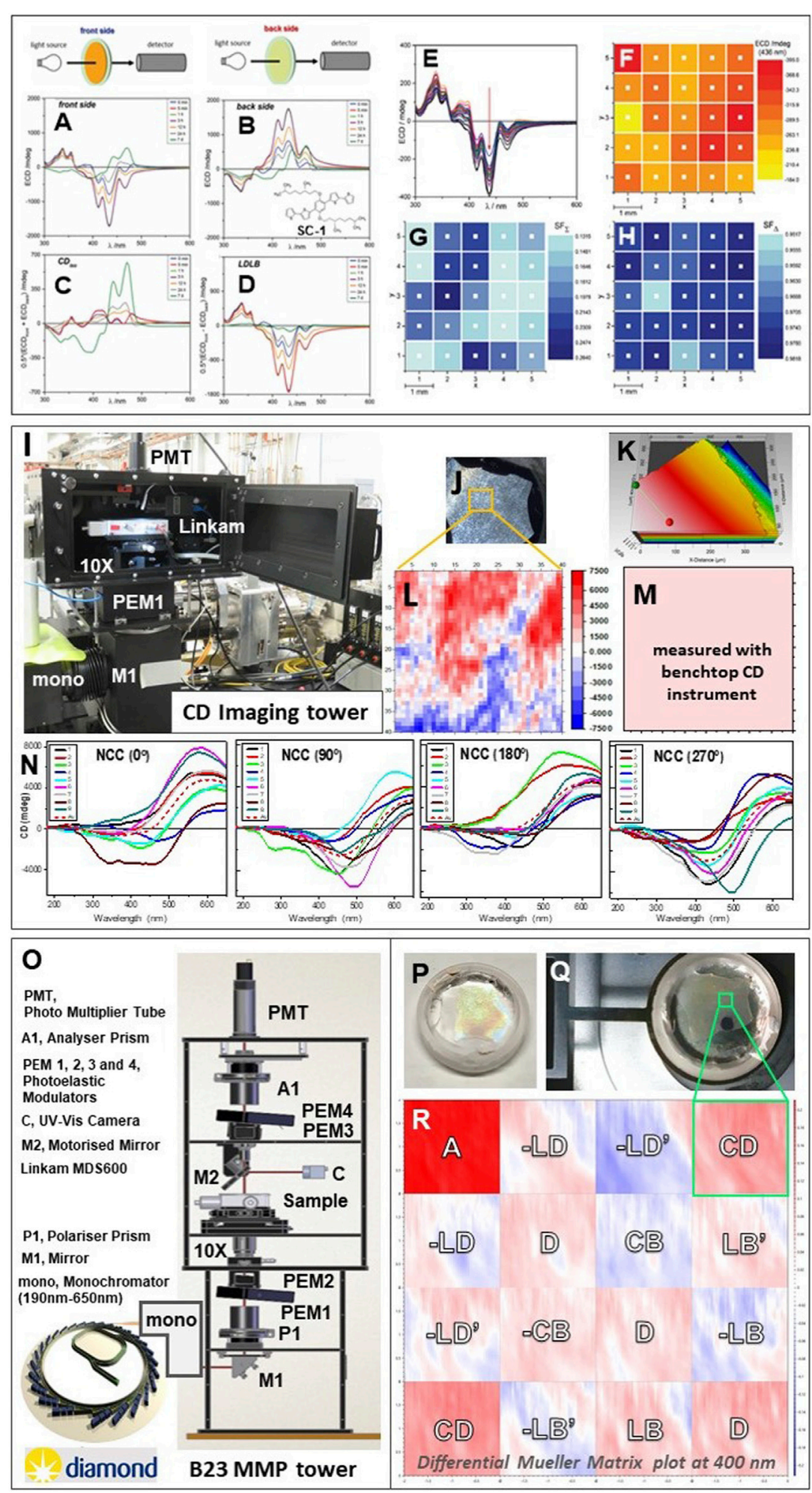

FIGURE 2 | (A) SRCD spectra of a thin film of $\pi$-conjugated chiral molecule spin coated (SC-1) on a fused silica subtrate measured on the front side orientation as illustrated by the cartoon. (B) SRCD spectra of back side orientations. (C) Calculated SRCD spectra termed $\mathrm{CD}_{\text {iso }}$ from the sum of spectra [(A + B)/2]. (D) Calculated SRCD spectra termed LDLB from the difference of spectra [(A-B)/2]. (E) $25 \mathrm{CD}$ spectra of front side orientation measured in a $5 \times 5$ grid at $1 \mathrm{~mm}$ intervals. (F) $2 \mathrm{D}$ map of SRCD intensities at $436 \mathrm{~nm}$ of the $5 \times 5$ grid measured in 2E. (G) Calculated 2D map of the $\mathrm{CD}_{\text {ISo }}$ intensities at $436 \mathrm{~nm}$ of the same $5 \times 5$ grid illustrated in $\mathrm{F}$ (the back side orientation is not shown). (H) Calculated 2D map of LDLB intensities at $436 \mathrm{~nm}$ of the $5 \times 5$ grid. All figures redrawn from Albano et al. (2019). (I) CD imaging 
FIGURE 2 | tower (CDi) equipped with X-Y motorized Linkam temperature controlled stage MDS600 and a UV transparent $10 \times$ objective lens to map a chiral nematic thin film of NCC ((J) $90 \mu \mathrm{m}$ in thickness using a Profilm 3D profilometer (K). (L) B23 2D map of SRCD intensities of NCC front side orientation measured with a $41 \times 41$ grid at $50 \mu \mathrm{m}$ interval steps for a total of $2 \mathrm{~mm}^{2}$ area. Note the SRCD intensity range from $+7,500$ (red) to $-7,500$ (blue) mdeg. (M) $2 \mathrm{D}$ map of CD intensity at $480 \mathrm{~nm}$ for the same area of $4 \mathrm{~mm}^{2}$ measured with Chirascan Plus CD instrument (Applied PhotoPhysics Ltd., United Kingdom). (N) SRCD spectra of NCC of a $3 \times 3 \mathrm{grid}$ at $1 \mathrm{~mm}$ interval steps (total area $2 \mathrm{~mm}^{2}$ ) as a function of rotation $\left(0,90,180\right.$, and $270^{\circ}$ ) around the axis normal to the sample surface (front side) and parallel to the propagation direction of the incident beamlight. (0) Optical elements of B23 MMP tower coupled to Diamond light source from bending magnet and B23 double grating subtracting monochromator, mono; M1, $45^{\circ}$ plane mirror; P1, linear polarizer; PEM1, PEM2, photoelastic modulators for polarization state generator; $10 \times$, objective UV transparent lenses; sample holder; M2, retractible mirror for UV camera; PEM3, PEM4, photoelastic modulators for polarization state analyzer; A1, linear polarizer; PMT,

photomultiplier tube detector. (P) Zoomed picture of the sample holder with NCC film specimen (Q). (R) Differential Mueller Matrix plot of the $2 \mathrm{~mm} \times 2 \mathrm{~mm}$ area with the 16 elements labeled for the corresponding physical interpretations: CD, circular dichroism; CB, circular birefringence; LD, horizontal linear dichroism; LB, horizontal linear birefringence; LD', 45 linear dichroism; LB', 45 linear birefringence; A, Absorbance; and D, Depolarization (D=A for materials with no depolarization). (Azzam, 1978).

supramolecular structure of the polymer. After 9 days of incubation, however, both front and back side were very similar in terms of profile shape and sign indicating a substantial reduction in LD and LB contributions. This can be better visualized with the sum of the SRCD spectra of the front and back side orientations divided by 2 that corresponds to the $\mathrm{CD}$ and $\mathrm{CB}$ contributions (Figure 2C) while the difference divided by 2 corresponds to the LD and LB elements (Albano et al., 2019) (Figure 2D).

For solutions, the solvent is the isotropic component whereas the solute being chiral is the anisotropic component that generates the CD. However, for solutions with macroscopic molecules such as large polymers and solid state films, CD, $\mathrm{CB}, \mathrm{LD}$, and $\mathrm{LB}$ contributions can be present and must be analyzed quantitatively with the differential Mueller matrix of data measured with MMP (Kuroda et al., 2000; Arteaga et al., 2012). In this paper the Mueller Matrix Ellipsometry is not discussed.

\section{DISCUSSION}

For thin films of solid state chiral materials, it is important to remember that all the polarization contributions are additive, and even without $\mathrm{LD}$ and $\mathrm{LB}$, the remaining $\mathrm{CB}$ contribution will affect the spectral shape hence its accurate interpretation in CD terms.

CD measurements carried out as a function of rotation around the axis normal to the sample surface and parallel to the incident light will show spectral differences if LD and LB contributions are present.

Nanocrystalline cellulose (NNC) obtained from the hydrolysis of cotton-derived cellulose can form chiral nematic liquid crystalline phases with a left helical orientation forming iridescent chiral nematic films upon drying. The SRCD spectra of a $3 \times 3$ grid of $1 \mathrm{~mm}$ interval steps of a thin film of NCC of about $90 \mu \mathrm{m}$ thick (Figure $2 \mathbf{K}$ ) measured as a function of rotations at $0,90,180$, and $270^{\circ}$ in the B23 vertical chamber equipped with a temperature controlled motorized $\mathrm{X}-\mathrm{Y}$ linear stage (Linkam MDS600) (Figure 2I) revealed unambiguosly the presence of LD and LB contributions as substantial spectral differences were observed (Figure $\mathbf{2 N}$ ). The same area of $4 \mathrm{~mm}^{2}$ of the $3 \times 3$ grid at $1 \mathrm{~mm}$ intervals was re-scanned as a $41 \times 41$ grid of $50 \mu \mathrm{m}$ spatial resolution revealing a $2 \mathrm{D}$ image with spectral intensity from $+7,500$ to $-7,500$ mdeg (Figure $2 \mathbf{L}$ ). It is important to notice that using a bespoke benchtop CD instrument (Chirascan Plus, APL, United Kingdom), the measured $C D$ illustrated in Figure $\mathbf{2 M}$ was the average of the same $4 \mathrm{~mm}^{2}$ area that did not reveal any of the complexity of the chiroptical properties of the NCC sample of Figure 2L. Of course the $2 \mathrm{D}$ image measured with B23 in imaging mode (Figure 2L) is certainly containing all the four polarization contributions, $\mathrm{CD}, \mathrm{CB}, \mathrm{LD}$, and $\mathrm{LB}$, for which only the use of a Mueller Matrix Polarimeter (MMP) (Arteaga et al., 2012) would enable the extraction of all those components.

The preliminary differential Mueller matrix plot of the nanocrystalline cellulose (NCC) obtained from the hydrolysis of cotton-derived cellulose (Figures 2P,Q) measured with the new B23 MMP tower for imaging facility (Figure 2O) confirmed that indeed all polarization contributions, $\mathrm{LD}, \mathrm{LB}, \mathrm{CD}$, and $\mathrm{CB}$ were present in the 2D CDi plot illustrated in Figure $2 \mathbf{L}$ with the use of B23 CDi tower. The CD elements of the differential Mueller Matrix were analyzed following the method developed by Arteaga of Kahr's group (Arteaga et al., 2012) and Ossikovski group (Arwin et al., 2016). Although the two elements in the antidiagonal representing the $\mathrm{CD}$ maps (Figure 2R) were both homogeneous in terms of uniform red colour associated to positive $\mathrm{CD}$, the intensity magnitudes were slightly different due to depolarization (Azzam, 1978). In this case, the average between the two CD elements of the differential Mueller Matrix should be considered revealing that the same area size of $2 \mathrm{~mm} \mathrm{x}$ $2 \mathrm{~mm}$ of the NCC thin film was indeed homogeneous unlike that observed with the CD imaging tower (Figure 2I) that appeared very heterogeneous (negative values in blue and positive in red (Figure 2L)). The MMP data (Figure 2R) confirmed the presence of the other CB, LD and LB components. This article is not meant to be a full characterisation of thin films of NCC that are known to have complex levels of mesoscale organisation but rather to raise the attention that, for chiral films, the use of differential Mueller Matrix is absolutely necessary in order to achieve a correct spectroscopic data interpretation. In solution, for instance, the conformation of proteins can be tuned by varying the solvent environment, $\mathrm{pH}$, temperature, surfactants, ligands, and concentration and one can assess by CD spectroscopy how big these changes are and how to optimise these parameters in order to achieve reproducible protein formulations (Siligardi and Hussain, 2015). This is also occurring in the solid state, however, the chiroptical spectroscopic assessment of the supramolecular structure to be optimise because it is related to its properties, like for optoelectronic materials where a homogeneous helical 
arrangement of the polymer maximises the charge transfer hence the production of current (Naaman et al., 2019), would be hampered and distorted by the presence of $\mathrm{CB}, \mathrm{LD}$ and $\mathrm{LB}$ contributions. That is why the use of the MMP to extract the $\mathrm{CD}$ information from the other optical properties of the solid state film is a must.

In here we have described the evolution of Diamond B23 beamline for synchrotron radiation circular dichroism from a powerful light source to push the wavelength boundaries of $\mathrm{Xe}$ lamps to the vacum UV spectral region to chiroptical imaging at higher spatial resolution. The new B23 MMP imaging facility (Figure 2O) is the latest tool that enables to see, and hopefully to control the chiroptical properties of materials of which properties rely on their homogeneous supramolecular structure. This will facilitate the identification of the parameters that can optimize such a homogeneity.

\section{INSTRUMENTATION}

The SRCD measurements were conducted using two types of B23 vertical sample chambers called towers depending upon the type and size on the sample specimens (Hussain et al., 2012; Hussain et al., 2016). One is equipped with a large $X-Y$ motorized linear stage that can accommodate large samples (maximum $12 \times 8 \mathrm{~cm}^{2}$ ) operating at room temperature, and the other with an X-Y motorized and temperature controlled Linkam MDS600 stage (for a maximum of $1.5 \mathrm{~cm} \times 1.5 \mathrm{~cm}$ sample area). The samples were held with appropriate $3 \mathrm{D}$ printed sample holders when necessary.

The MMP built by Hinds Instruments (United States) coupled to Diamond B23 light source and the double grating subtractive monochromator (Olis Instruments, United States) operating in the $190-750 \mathrm{~nm}$ region is composed of four photoelastic modulators operating simultaneously at different frequencies, two in the polarization state generator and two in the polarization state analyser (Figure 20). The data acquisition and data processing were implemented by Hinds Instruments following the method described in Arteaga et al. (2012).

The MMP was calibrated using aqueous solution of $1 \mathrm{~S}(+) 10-$ Camphorsulfonic acid $(5 \mathrm{mg} / \mathrm{ml})$ in a $1 \mathrm{~cm}$ pathlength cuvette to give a positive CD intensity at $290.5 \mathrm{~nm}$ of about $1500 \mathrm{mdeg}$. The other polarization elements of the differential Mueller Matrix were calibrated using a thin film of an optoelectronic material (F8BT:aza [P]) characterised by Mueller Matrix Ellpsometry using a Woollam RC2 (model DI) instrument and reported in Wade et al. (2020). In Wade at al. (2020), the homogeneity of the

\section{REFERENCES}

Albano, G., Gorecki, M., Pescitelli, G., Di Bari, L., Javorfi, T., Hussain, R., et al. (2019). Electronic circular dichroism imaging (CDi) maps local aggregation modes in thin films of chiral oligothiophenes. New J. Chem. 43, 14584-14593. doi:10.1039/c9nj02746g

Arago, D. F. J. (1811). "Memoir on a remarkable modification experienced by light rays in their passage through certain diaphanous bodies, and on some other new optical phenomena," in Mémoires de la classe des sciences mathématiques et physiques de l'Institut Impérial de France. 1st part. Paris, 93.-134. supramolecular structure of the thin film of F8BT:aza $[\mathrm{P}]$ was assessed at 50 micron spatial resolution by CDi using the B23 vertical chamber of Figure 2I as the size of the collimated light beam of the Woollam RC2 ellipsometer was about 3-4 mm in diameter.

The CD spectrum from which Figure $\mathbf{2} \mathbf{M}$ was generated was measured with Chirascan Plus (Applied Photophysiscs, United Kingdom). The thickness of the thin film of NCC specimen in Figure $\mathbf{2 K}$ was measured with the Profilm 3D profilometer (Filmetrics, United States). The MMP was calibrated using aqueous solution of $1 \mathrm{~S}$ (+) 10 Camphorsulfonic acid $(5 \mathrm{mg} / \mathrm{ml})$ in a $1 \mathrm{~cm}$ pathlength cuvette to give a positive CD intensity at $290.5 \mathrm{~nm}$ of about 1500 mdeg. The other polarization elements of the differential Mueller Matrix were calibrated using a thin film of an optoelectronic material (F8BT:aza [P]) characterised by Mueller Matrix Ellpsometry using a Woollam RC2 (model DI) instrument and reported in Wade et al. (2020). In Wade at al. (2020), the homogeneity of the supramolecular structure of the thin film of F8BT:aza [P] was assessed at 50 micron spatial resolution by CDi using the B23 vertical chamber of Figure 2I as the size of the collimated light beam of the Woollam RC2 ellipsometer was about 3-4 mm in diameter.

\section{DATA AVAILABILITY STATEMENT}

The original contributions presented in the study are included in the article/Supplementary Material, further inquiries can be directed to the corresponding author.

\section{AUTHOR CONTRIBUTIONS}

GS conceived the study and wrote the paper. TJ, RH, and GS developed the CD imaging measurement technique. TJ, and GS performed the MMP spectral measurements. All authors discussed the results.

\section{ACKNOWLEDGMENTS}

We would like to thank Prof M. Tiritici of ICL for NCC sample and J. Freudenthal of Hinds Intruments (Hillsboro, Oregon, United States) for his help in calibrating the B23 MMP instrument during B23 beamtime commissioning (CM26447-1).

Arteaga, O., Freudenthal, J., Wang, B., and Kahr, B. (2012). Mueller matrix polarimetry with four photoelastic modulators: theory and calibration. Appl. Opt. 51, 6805-6817. doi:10.1364/ao.51.006805

Arwin, R., Mendoza-Galvan, A., Magnusson, R., Andersson, A., Landin, J., Järrendahl, K., et al. (2016). Structural circular birefringence and dichroism quantified by differential decomposition of spectroscopic transmission Mueller matrices from Cetonia aurata. Opt. Lett. 41, 3293-3296. doi:10.1364/ol.41. 003293

Azzam, R.M.A. (1978). Propagation of partially polarized light through anisotropic media with or without depolarization: A differential 4 X 4 matrix calculus. J. Opt. Soc. Am., 68, 1756-1767. 
Biot, J. B. (1815). Phénomènes de polarisation successive, observés dans des fluides homogènes. Bull. Sci. Soc. Philomath., 190-192.

Borisenko, K. B., Shanmugam, J., Williams, B. A., Ewart, P., Gholipour, B., Hewak, D. W., et al. (2015). Photo-induced optical activity in phase-changed memory materials. Sci. Rep. 1-5, 8770. doi:10.1038/srep08770

Cotton, A. C. (1895). Absorption inegale des rayons circulaires. R. Acad. Sci. Paris. 120, 989-991.

Fresnel, A. (1824). Considérations théoriques sur la polarisation de la lumière. Bull. Sci. Soc. Philomath., 23, 147-158.

Gerdova, A. (2013). Absorption flattening correction of circular dichroism spectra of particle suspensions. Master's thesis. Scotland (United Kingdom: University of Strathclyde.

Grosjean, M., and Legrand, M. (1960). C R Hebd. Seances Acad. Sci. 251, 2150.

Hussain, R., Javorfi, T., and Siligardi, G. (2016). High-throughput SRCD using multi-well plates and its applications. Sci. Rep. 6, 1-6. doi:10.1038/srep38028

Hussain, R., Javorfi, T., and Siligardi, G. (2012). Circular dichroism beamline B23 at the diamond light source. J. Syn. Rad. 19, 132-135. doi:10.1107/ S0909049511038982

Kuroda, R., Berova, N., Nakanishi, K., and Woody, R. W. (2000). "Solid-state CD: application to inorganic and organic chemistry," in Circular dichroism: principles and applications. 2nd Edn Wiley-VCH, New York, 912.

Lowry, T. M. (1935). Optical rotatory power. J. Soc. Chem. Ind. 54, 782. doi:10. $1002 /$ jctb. 5000543410

Mason, S. F. (1978). "Optical activity and chiral discrimination," in Proceedings of the NATO advanced study institute, Falmer, England, September 10-22, 1978. Editor S. F. Mason (Falmer, England: University of Sussex), 11-24.

Naaman, R., Paltiel, Y., and Waldeck, D. H. (2019). Chiral molecules and the electron spin. Nature Reviews Chemistry 3, 250-260.
Pasteur, L. (1849). Relationship which may exist between the crystalline form and the chemical composition and on the cause of rotational polarization. C. R. Acad. Sci. Paris 26, 535-538.

Siligardi, G., and Hussain, R. (2015). CD Spectroscopy: An Essential Tool in Quality Control of Protein Folding. Structural Proteomics: High-Throughput Methods, Methods Mol. Biol. (Owens, R.J., ed), Springer NY, 1261, 255-276.

Sitsanidis, E. D., Piras, C. C., Alexander, B. D., Siligardi, G., Javorfi, T., Hall, A. J., et al. (2018). Circular dichroism studies of low molecular weight hydrogelators: the use of SRCD and addressing practical issues. Chirality 30 (15), 708-718. doi:10.1002/chir.22850

Velluz, L., Legrand, M., and Grosjean, M. (1965). Optical circular dichroism. London, New York, NY: Verlag Chemie, 247.

Wade, J., Hilfiker, J. N., Brandt, J. R., et al. (2020). Natural optical activity as the origin of the large chiroptical properties in p-conjugated polymer thin films. Nature Communications 11, 6137-6148.

Zinna, F., Resta, C., Gorecki, M., Pescitelli, G., Di Bari, L., Javorfi, T., et al. (2017). Circular dichroism imaging: mapping the local supramolecular order in thin films of chiral functional polymers. Macromolecules 50, 2054-2060. doi:10. 1021/acs.macromol.6b02590

Conflict of Interest: RH, TJ, and GS were employed by Diamond Light Source Ltd.

Copyright (C) 2021 Hussain, Jávorfi and Siligardi. This is an open-access article distributed under the terms of the Creative Commons Attribution License (CC BY). The use, distribution or reproduction in other forums is permitted, provided the original author(s) and the copyright owner(s) are credited and that the original publication in this journal is cited, in accordance with accepted academic practice. No use, distribution or reproduction is permitted which does not comply with these terms. 